\title{
Impact of metal removal features in the reverse zones on shaping of the machined surface in the process of cylindrical grinding with traverse feed
}

\author{
Pavel Pereverzev ${ }^{1}$, Aleksandra Akintseva ${ }^{1,{ }^{*}}$, and Masar Alsigar ${ }^{1}$ \\ ${ }^{1}$ South Ural State University, Department of Engineering and Technology, 454080 Chelyabinsk, \\ Lenin Avenue 76, Russian Federation
}

\begin{abstract}
This paper presents a model of the formation process of surface zones of external cylindrical grinding with traverse feed at a computer numerical control machine $(\mathrm{CNC})$. This article, have investigated modelling features of the grinding process in reverse zones. On the basis of the model of grinding surface formation a detailed step by step calculations of the current values of the actual radial feed and radius dimension in different areas of a treated surface is presented. The model allows maintaining calculations with presence of the initial radial runout of the workpiece for estimated automatic cycle of radial and axial feeds changing and other technological parameters.
\end{abstract}

\section{Introduction}

At present in the field of automated manufacturing and industrial automation there are no models, methods and soft ware which allow to forecast the accuracy of the parts processing during cylindrical grinding with traverse feed on CNC machines. Productivity management is carried out by the simultaneous and joint control of automatic step cycles of the radial and axial feeds according to the commands of the active control device depending of the allowance remaining part. Therefore, grinding $\mathrm{CNC}$ machines are used ineffectively because of a underrating of cutting modes during manual selection of cycles in radial and axial feed.

Thus, the aim of work is to increase the efficiency of use the cylindrical grinding machines with $\mathrm{CNC}$ by designing models and method which allow to make an analytical calculation of the depth cut actual values and forecast the change of radius dimensions of the treated surface in reverse and non-reverse areas under the influence of various variable factors and grinding conditions.

In order to achieve the goal, the following modifications have been completed:

- developing an analytical model of surface formation on the operation of cylindrical grinding with axial feeds, when controlling the operation by using automatic cycles in radial and axial feed;

\footnotetext{
*Corresponding author: akintsevaav@susu.ru
} 
- presenting a method of calculation for radial actual values of a treated surface throughout the whole grinding process, on the basis of the obtained model of grinding surface formation

A literature review revealed that many scientists have been engaged in studying, modelling and simulating of grinding processes and automatic cycles of operational performance management, some examples of scientists S. Malkin [1], Lurie G.B. [2], Maslov E.N. [3], Morgan, M.N., Cai, R., Guidotti, [4] A., Allanson, D.R., Rowe, W.B. [7], Bratan S.M. [5], Novoselov YU.K. [6]. However, there is no information about researches in the field of formation surface modeling in the grinding process with automatic control cycle of radial and axial feeds $[7,8,11,12,13,18]$.

Therefore, we can conclude that, despite the numerous studies and enormous effort in theoretical and practical basis to study modeling field of the grinding surface, until now the problem of developing a model for the formation of a surface by automatic control cycle with radial and axial feeds is not solved in reverse and non-reversible zones of external cylindrical grinding. The absence of surface formation model makes it impossible to solve the further task of calculating the optimal grinding cycles of radial and axial feeds.

\section{Effects of metal removal on the reverse zone of shaping of the machined surface of cylindrical grinding process with traverse feed}

Let's consider features of cylindrical grinding process in the automatic cycle of radial and axial feeds controlling in Figure.1.

Grinding cycle in the section 1 consists of five stages S1-S5 (Fig. 1). S1 CG (cylindrical grinding with radial feed) is performed in the cylindrical infeed grinding mode with radial feed $S_{n}$. Grinding pastern of the wheel goes on the program feed value tn which is equal to a given value of the cutting depth on the double stroke (DS). Due to the wheel overtravel, the grinding is made by a part of the wheel height. On the Fig. 1 on S1 a graph of the program and actual feeds in the cylindrical grinding with radial feed is shown $[2,5,6]$.

$\mathrm{S} 2$ is conducted in the cylindrical infeed grinding mode in combination with a dwelling stage, radial feed is equal to 0 . On this stage the wheel with radial feed stops, axial feed $S_{n p}$ turns on. Grinding is carried out due to the preload arising on the S1. On the Fig. 1 on S2 the graph of the program and actual feeds in cylindrical grinding with radial feed is shown. $\mathrm{S} 3$ is carried out in the cylindrical grinding with axial feed (CGAF). Grinding wheel is deepen into the radial program feed value $\Delta t_{n k, i, z}$; due to the presenceof initial radial beating of the workpiece is calculated on each $k$-th radius of the part, $i$-th turn of the part and $z$-th stage of the grinding cycle. On this stage axial feed $S_{n p}[\mathrm{~mm} / \mathrm{min}]$ turns on and the working stroke (WS) begins. As it is another grinding type (CGAF), actual feed $\Delta t_{\phi k, i, z}$ has another physical meaning and it is measured in $\mathrm{mm} / \mathrm{stroke}$ or $\mathrm{mm} / \mathrm{turn}$. Graph of $\Delta t_{\phi k, i, z}$ is constantly changing and shown as a broken line. On Fig. 1a the graph of feeds $\Delta t_{\phi}$ on S3 adjoins graphs on S1 and S2. On this stage the axial program feed is greater than 0 (Fig. $1 b)$.

S4 is carried out in CGAF mode but on reverse idling stroke (IS) when there is no additional radial feed. On this stage the axial feed $S_{n p}$ for IS is turned on and it is greater than 0 (Fig. 1c).

S5 is carried out in the cylindrical infeed grinding mode with dwelling time, radial feed is equal to 0 . On this stage wheel with axial feed stops and the radial feed $S_{n}$ turns on. Surface grinding is carried out due to the remaining preload after S4. On Fig. 1b,c computed pattern is shown; this pattern shows interrelations between graphs of accumulated program $t_{n k, i, z}$ and actual $t_{\phi k, i, z}$ feeds, preload $t_{t k, i, z}$, elastic deformation $y_{k, i, z}$ of 
the technological system with radiuses of the treated surface $R_{k, i, z}$ in the process of grinding noncircular workpiece with maximal radius $R_{\text {загmax }}$, initial radiuses $R_{\text {заг }}$ of the workpiece and initial radial beating which is calculated by the formula:

$$
\Delta R_{\text {загk }}=R_{\text {загmax }}-R_{3 a z k}
$$

a)

b)

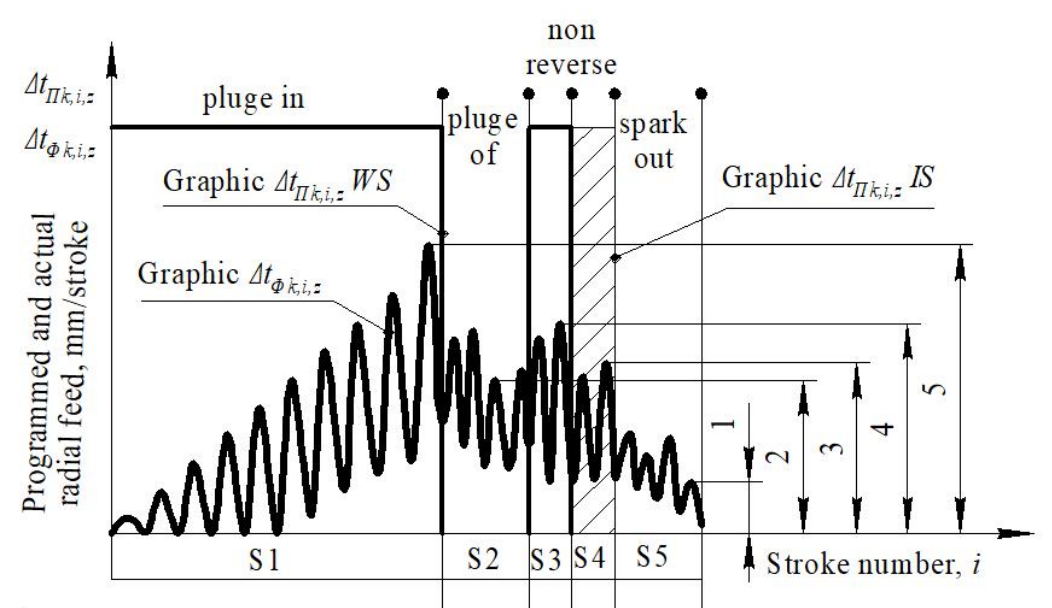

c)

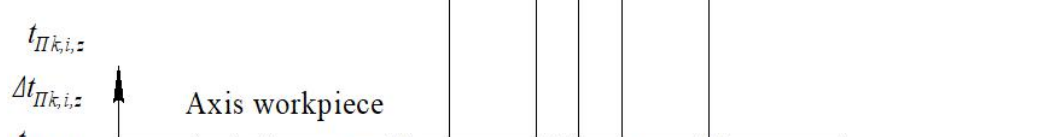

)

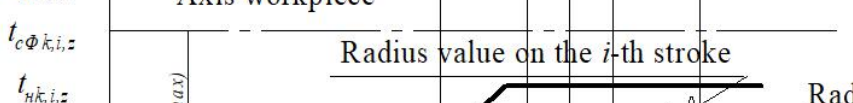

Radius value on the $i-1$ stroke

II

)

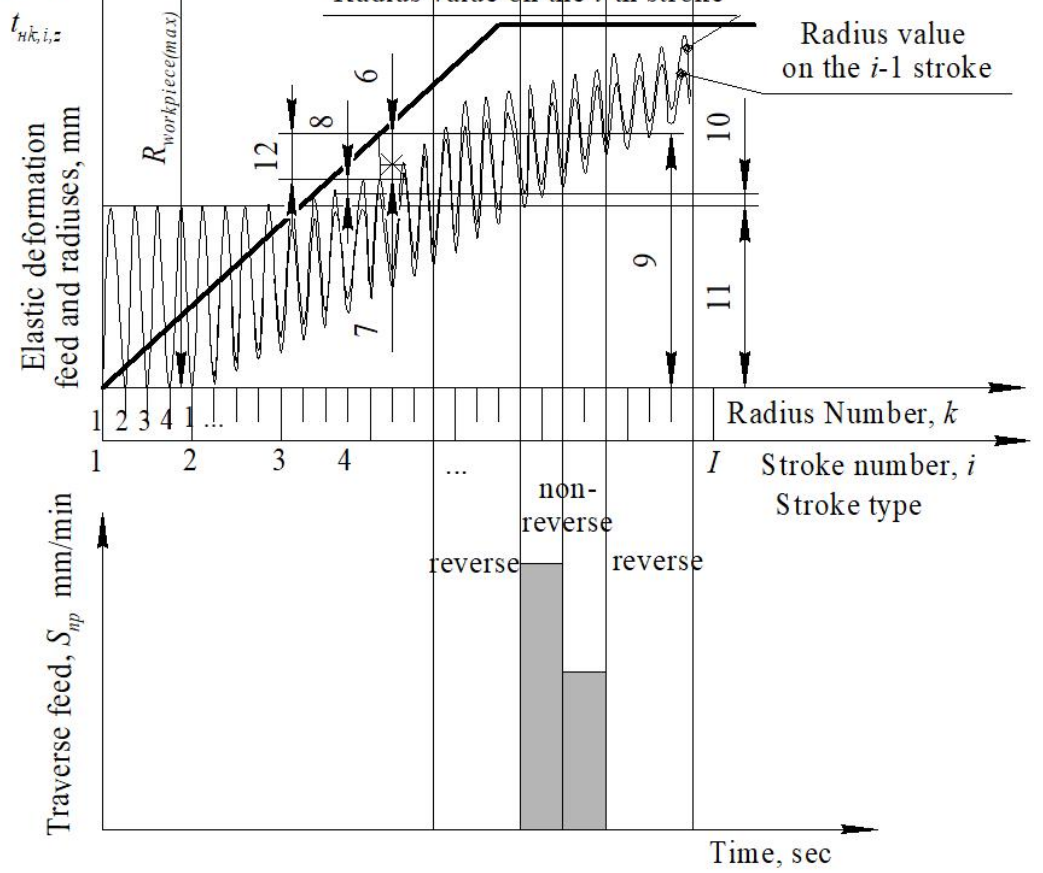

Fig. 1. The relationship between elastic deformations of the technological system with program and actual feeds (entry-section): IS - idling stroke; WS - work stroke; 1 - change value at $\Delta t_{\phi k, i, z}$ (S5); 2 - change value at $\Delta t_{\phi k, i, z}(\mathrm{~S} 4) ; 3$ - change value at $\Delta t_{\phi k, i, z}(\mathrm{~S} 3) ; 4$ - change value at $\Delta t_{\phi k, i, z}(\mathrm{~S} 2) ; 5$ - 
change value at $\Delta t_{\phi k, i, z}(\mathrm{~S} 1) ; 6-\Delta t_{\Pi k, i, z} ; 7-y_{k, i, z} ; 8-\Delta t_{\Phi k, i, z} ; 9-t_{\Pi k, i, z} ; 10-t_{\phi k, i, z} ; 11-\Delta R_{3 a z k} ; 12$ $-t_{H k, i, z}$.

According to computed pattern on Fig. 1, values of accumulated program $t_{n k, i, z}$ and actual $t_{\phi k, i, z}$ feeds are calculated as a sum of feeds on each stroke of the wheel.

$$
\begin{aligned}
t n_{k, i, z} & =\sum_{l}^{k} \sum_{l}^{i} \sum_{l}^{z} \Delta t_{n_{k, i, z^{\prime}}} \\
t \phi_{k, i, z} & =\sum_{l}^{k} \sum_{l}^{i-1} \sum_{l}^{z} \Delta t_{\phi_{k, i, z}} .
\end{aligned}
$$

It gives an opportunity to calculate a form change of the grinding surface and to forecast a processing error at the end of the grinding cycle.

Considering the fact that radial component of the cutting force also depends on the actual feed $[9,10,14,15,16]$, this interrelation can be expressed by the following formula:

$$
P_{y k, i, z}=K_{1} \Delta t_{\phi k, i, z}+K_{2} \sqrt{\Delta t_{\phi k, i, z}},
$$

$K_{1}$ and $K_{2}$ are analytical coefficient, characterizing interrelation between different technological parameters of the grinding process [17, 19, 20, 21, 22, 24].

Let us estimate the interrelation between program and actual feeds through elastic deformations of the technological system. Grinding process is carried out due to the preload $\Delta t_{H k, i, z}$ in the technological system which, according to Fig. 1, is equal to sum of elastic deformation $y_{k, i, z}$ and actual feed $\Delta t_{\phi k, i, z}$.

$$
\Delta t_{\mu k, i, z}=y_{k, i, z}+\Delta t_{\phi k, i, z}
$$

Dimensional relations on Fig. 1 show that actual preload value $t_{t k, i, z}$ can be calculated through the accumulated program feed $t_{n k, i, z}$, actual feed $\Delta t_{\phi k, i, z}$ and initial radial beating $\Delta R_{k}$ of the workpiece by the following formula:

$$
\begin{gathered}
\Delta t_{H k, i, z}=\Delta t_{\Pi k, i, z}-\Delta t_{\phi k, i, z}-\Delta R_{k}, \\
\Delta R_{k}=R_{\max }-R_{W k} .
\end{gathered}
$$

$R_{\max }$ is a maximal radius of the workpiece.

Solving complex of formulas (1) - (7) about actual feed $\Delta t_{\phi k, i, z}$, the following formula (8), which allows to calculate actual values of the actual feed $\Delta t_{\phi k, i, z}$ on each turn of the wheel during the whole grinding cycle $[9,10,22,23]$ could be got:

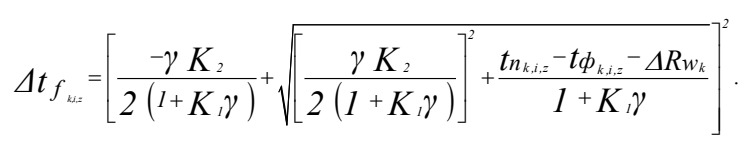

Actual values of the actual feed $\Delta t_{\phi k, i, z}$ got through the formula (8) let us calculate actual values of radiuses $R_{k, i, z}$ of the treated surface, cutting force and elastic deformations by substitution $\Delta t_{\phi k, i, z}$ into formulas (2) - (5).

\section{Conclusion}

1) There are no optimization systems providing a required accuracy and quality of the treated surface with fluctuating conditions of the processing, which appear during 
workpiece batch processing in automated engineering for cylindrical grinding operations with traverse feed on CNC machines;

2) Model of surface formation has a wide variety of technological factors and has an analytical character, because it has got on the basis of mathematical interrelation between radiuses dimensions of the treated surface and actual feeds with analytical model of cutting force, which is designed on the basis of fundamental regularities of the cutting process mechanics and theory of metal plastic deformation in the cutting area. This model estimates relation with main technological factors:

- physical and mechanical properties of grinded metal (stress intensity);

- geometric parameters of the contact area of the wheel and the workpiece (actual feed speed, workpiece and wheel diameters, workpiece rotation frequency, width of the workpiece treated surface);

- elastic properties of the technological system (elastic deformations and compliance of the technological system);

- grinding wheel characteristics and grade of wheel grains blunting.

3) Interrelation between the model of allowance removal with cutting force gives an opportunity to estimate the influence of different technological factors on productivity, accuracy and quality of processing during variation of the control parameters of the cylindrical exterior grinding cycle. These parameters are cycle stage quantity, program speeds of radial speed on each cycle stage, distribution of allowance on cycle stages.

\section{References}

1. S. Malkin, C. Guo, Grinding Technology: Theory and Applications of Machining with Abrasives (2008)

2. G.B Lur'e, Splifovanie metallov (1969)

3. E.N. Maslov, Teoriya shlifovaniya materialov (1974)

4. M.N. Morgan, R. Cai, A. Guidotti, D.R. Allanson, W.B. Rowe, Machining Science and Technology 10 (2), 157 (2006)

5. S. Bratan, S. Roshchupkin, A. Kolesov, B. Bogutsky, Identification of removal parameters at combined grinding of conductive ceramic materials, MATEC Web of Conferences, V. 129, 01079 (2017)

6. Y.K. Novoselov, Dynamics of surface formation during abrasive processing (2012)

7. W.B. Rowe, S.C. Black, B. Mills, H.S. Qi, M.N. Morgan, CIRP Ann Manuf-Technol 44 (1), 329 (1995)

8. S. Malkin, N.H. Cook, ASME J Eng Ind 93, 1120 (1971)

9. P.P. Pereverzev, Bulletin of the South Ural State University. Series «Mechanical Engineering» 12 (271), 152 (2012)

10. P.P. Pereverzev, M.K. Alsigar, International scientific publication «Modern Fundamental and Applied Research» 4 (27), 31 (2017)

11. S. Kannapan, S. Malkin, ASME J Eng Ind 94, 833 (1972)

12. L. Lichun, F. Jizai, J. Peklenik, CIRP Ann Manuf-Technol 29(1), 245 (1980)

13. A.S. Lavine, S. Malkin, T.C. Jen CIRP Ann Manuf-Technol 38(1), 557 (1989)

14. P.P. Pereverzev, M.K. Alsigar, Journal «METALLOOBRABOTKA» 6, 152 (2017)

15. P.P. Pereverzev., A.V. Akintseva, Russian Engineering Research 36 (11), 974 (2016)

16. P.P. Pereverzev, M.K. Alsigar, V International Correspondence Scientific and Practical Conference «Automated Design in Mechanical Engineering» 29, 42 (2017) 
17. A.V. Akintseva, P.P. Pereverzev, MATEC Web of Conferences 129, 01019 (2017).

18. H.K. Toenshoff, J. Peters, I. Inasaki, . CIRP Ann Manuf Technol 41(2), 677 (1992)

19. P.P. Pereverzev, A.V. Akintseva, STIN 4, 23 (2016)

20. P.P. Pereverzev, A.V. Akintseva, Procedia Engineering 129, 121 (2015)

21. P.P. Pereverzev, A.V. Akintseva, Herald of SUSU. Series «Mechanical Engineering» 2, 61 (2016)

22. P.P. Pereverzev, M.K. Alsigar, II International Scientific and Practical Conference «Mechatronics, Automation and Robotics» 22, 124 (2018)

23. A.V. Akintseva, P.P. Pereverzev, MATEC Web of Conferences 129, 01018 (23017)

24. A.V. Akintseva, M.K. Alsigar, The future of machine building in Russia: conf. young scientists and specialists. 17, 19 (2017) 